\title{
Sorbitol dehydrogenase expression is regulated by androgens in the human prostate
}

\author{
ZOLTÁN SZABÓ $^{1}$, JENNI HÄMÄLÄINEN ${ }^{1}$, ILDIKÓ LOIKKANEN ${ }^{1}$, ANNE-MARI MOILANEN ${ }^{1}$, \\ PASI HIRVIKOSKI ${ }^{2}$, TIMO VÄISÄNEN ${ }^{1,2}$, TIMO K. PAAVONEN ${ }^{4}$ and MARKKU H. VAARALA ${ }^{1,3}$ \\ ${ }^{1}$ Department of Pathology, University of Oulu; ${ }^{2}$ Department of Pathology and ${ }^{3}$ Division of Urology, \\ Department of Surgery, Oulu University Hospital, Oulu; ${ }^{4}$ Department of Pathology, \\ University of Tampere and Tampere University Hospital, Tampere, Finland
}

Received December 1, 2009; Accepted February 12, 2010

DOI: $10.3892 /$ or_00000755

\begin{abstract}
Sorbitol is an intermediate in the polyol pathway, which converts from glucose to fructose by sorbitol dehydrogenase (SORD). Androgens are essential for the development of prostate cancer. We studied castration-induced gene expression changes in the human prostate using the GeneChip array, and identified SORD as being androgen-regulated in the human prostate. A putative androgen-responsive regulatory region at the SORD 5' promoter was identified using promoter deletion constructs in a luciferase reporter assay in COS-7 cells. Chromatin immunoprecipitation assay was used to assess the binding of androgen receptor to suggested androgen responsive regulatory region. Finally, the expression of SORD in the human prostate was evaluated in 29 prostate tissue samples by immunohistochemistry. The expression of SORD decreased after castration. Androgen supplementation to the LNCaP prostate cancer cell line led to a 7.5-fold increase in SORD mRNA expression. Furthermore, a chromatin immunoprecipitation assay proved that the androgen receptor can bind to this putative androgen-responsive regulatory region. Finally, the expression of SORD in the human prostate was localised to epithelial cells of both benign and malignant prostate tissue by immunohistochemistry. In prostate cancer, increased immunostaining was associated with high Gleason patterns and high serum prostate-specific antigen concentrations. These results show that SORD is a novel androgen-regulated gene in the human prostate and suggest the need for more detailed analysis of the physiological role of SORD in the prostate.
\end{abstract}

\section{Introduction}

Sorbitol dehydrogenase (SORD) catalyses the interconversion of polyols and their corresponding ketoses. SORD deficiency

Correspondence to: Dr Markku H. Vaarala, Division of Urology, Department of Surgery, Oulu University Hospital, PO Box 21, Oulu, FIN-90029 OYS, Finland

E-mail:markku.vaarala@oulu.fi

Key words: sorbitol dehydrogenase, prostate cancer, androgen regulation has been linked with congenital cataracts (1). SORD expression is high in the human kidney and lens of the eye (2) and widely expressed in other human tissues as well (3). The clinical significance of SORD has been evaluated mainly with a focus on the complications of diabetes (4). In humans, SORD is secreted by the epididymal epithelium. This supports the suggestion that the polyol pathway plays a role in human sperm physiology (5). Androgens are a prerequisite for the development of prostate cancer, which is the most common malignancy of men in the western world. Androgen ablation triggers cell death or cell cycle arrest of prostate cancer cells (6). The action of androgens on gene expression is mediated via the androgen receptor (AR) (7). The AR belongs to a superfamily of nuclear receptors that function as ligand-activated transcription factors. The AR is a type 1 receptor that forms homodimers after ligand binding and binds inverted repeattype DNA response elements in the regulatory regions of the target genes (8).

Here, we used a GeneChip array to search for novel genes regulated by androgens in the human prostate. SORD was identified as being down-regulated by castration, indicating androgen-dependent expression. SORD androgen induction was also detected in the prostate-derived cell line LNCaP. We studied the expression and tissue localisation of SORD protein in the prostate. SORD immunostaining was detected in epithelial cells with increased staining intensity in samples with high Gleason patterns and high serum prostate-specific antigen (PSA) concentrations. We studied the mechanism of SORD regulation by androgens and present evidence showing that androgens may regulate SORD by the classical ARmediated mechanism, as the AR binds to the SORD 5' regulatory region. These results establish SORD as a novel androgenregulated enzyme in the human prostate with increased immunostaining in poorly differentiated prostate carcinomas.

\section{Materials and methods}

Patient samples and GeneChip protocol. Sample collection, preparation and GeneChip procedures have been described in detail elsewhere (9). Briefly, androgen-regulated genes in the human prostate were studied using prostate biopsy samples taken three days after surgical castration performed for the treatment of prostate cancer. RNA from 6 patient samples 
was individually labelled and used for the GeneChip array. Three samples were histologically confirmed as benign prostate tissue from radical prostatectomy specimens and 3 samples were from biopsies taken after surgical castration performed as a therapeutic procedure for prostate cancer. The expression profiles were analysed using Chipster software (http://chipster. csc.fi). All GeneChips were normalised using the RMA (robust multiarray average) method and gene expression intensity estimates were received in $\log 2$-transformed values. The archived prostate specimens were initially collected from patients that underwent prostatectomy or transurethral resection of the prostate for the treatment of prostate cancer or benign prostatic hyperplasia (BPH) at the Oulu University Hospital. The material consisted of 25 prostate cancer and four BPH tissue specimens. There were 12, 4 and 9 prostate cancer samples from radical prostatectomy, transurethral resection for symptoms of $\mathrm{BPH}$ (occult carcinoma) and palliative transurethral resections of prostate cancer patients with castration therapy ongoing, respectively. Different Gleason patterns were selected for the multitissue block. There were 6, 7, 6 and 6 samples with Gleason pattern 2, 3, 4 and 5 , respectively, and $4 \mathrm{BPH}$ samples. Gleason patterns were used in statistical analyses instead of the Gleason scores because the specific patterns were selected for the multitissue block. Information about the corresponding PSA concentrations preceding tissue sampling were obtained from patient records. The PSA concentrations preceding tissue sampling varied from 2.9 to $262 \mathrm{ng} / \mathrm{ml}$ for cases present at the multitissue block. Informed consent was obtained from every subject before fresh tissue sampling. The ethics council of the Northern Ostrobothnia Hospital District approved the protocol.

Cell culture. Prostate cancer cell line LNCaP (CRL-1740) cells and African green monkey kidney fibroblast-like cell line COS-7 (CRL-1651) cells were purchased from the American Type Culture Collection (ATCC, Manassas, VA, USA). LNCaP and COS-7 cell cultures were maintained in RPMI-1640 (Sigma-Aldrich, St. Louis, MO, USA) supplemented with $10 \mathrm{mM}$ HEPES, $1 \mathrm{mM}$ sodium pyruvate, $2.5 \mathrm{~g} / 1$ D-glucose or DMEM (Sigma-Aldrich) supplemented with $4500 \mathrm{mg} / 1$ glucose, L-glutamine and $1 \%$ penicillinstreptomycin (Invitrogen-Gibco, Carlsbad, CA, USA), respectively, and were added to $10 \%$ fetal bovine serum (HyClone, Logan, UT, USA) at $37^{\circ} \mathrm{C}$ in a humidified atmosphere of $5 \%$ $\mathrm{CO}_{2}$. Fetal bovine serum was substituted with charcoaltreated fetal bovine serum in the hormone-induction experiments. Seventy-two hours prior to experiments the LNCaP cells were plated with $1 \times 10^{6}$ cells per plate. Cells were treated with $10 \mathrm{nM}$ synthetic androgen R1881 (Perkin-Elmer, Boston, MA, USA) or an equal volume of ethanol for $0,6,24$ or $48 \mathrm{~h}$. After incubation, cells were harvested, washed with phosphatebuffered saline and used directly for isolation of RNA. For transfection, COS-7 cells were grown to $80 \%$ confluency before transfection.

Plasmids. The SORD (GenBank accession no. NM_003104.4) promoter fragments were cloned into pGL3-Basic (Promega, Madison, WI, USA) upstream of the luciferase reporter gene at the HindIII and SacI sites. A genomic clone (rzpdb737b102039d) was purchased from imaGenes (www. imagenes-bio.de) for use as a template for PCR with oligonucleotides 5'-GTACAAGCTTGGACGTTTGGTCGCT CTG-3' and 5'-CGAAGAGCTCTGCCCAGTTCAACCT TCC-3' containing HindIII and SacI digestion site sequences, respectively, at their 5'-ends to clone the SORD 5' upstream promoter region starting from position -2391 to +101 (positions numbered from the transcription initiation start site). This region corresponds to nucleotides 45312950-45315442 in NC_000015.9 (NCBI reference sequence). Deletion constructs were created respectively using oligonucleotides 5'-CGAAG AGCTCTGGTGTGCCATTTGCTAAGA-3', 5'-CGAAGAG CTCTAGGTAATCAAGCGGGCAAG-3' and 5'-CGAAGA GCTCGTAGCACCAACCAGATT-3' for the creation of 5' upstream deletion constructs $-2230,-1843$ or -1699 to +101 , respectively. All clones were sequenced to exclude mutations and deletions during cloning. Potential androgen response elements (AREs) were identified using the TESS (Transcription Element Search System) programme (http://www. cbil.upenn.edu/cgi-bin/tess/tess).

Transfections. The COS-7 cells were seeded into 24-well plates at 30000 cells per well $24 \mathrm{~h}$ before transfection. The cells were transfected with Dotap transfection reagent (Roche Diagnostics GmbH, Penzberg, Germany) according to the manufacturer's protocol, using $0.65 \mu \mathrm{g}$ of plasmid and $3 \mu \mathrm{g}$ of transfection reagent and DMEM media (SigmaAldrich). AR cDNA in pcDNA3.1 vector (a gift from Professor T. Visakorpi, University of Tampere, Tampere, Finland) was cotransfected. Twenty-four hours after transfection, the media was replaced with DMEM and $100 \mathrm{nM}$ R1881 (Perkin-Elmer) was added when appropriate for $48 \mathrm{~h}$. To provide an internal control for transfection efficiency, a second reporter plasmid pRL-TK (Promega) was transfected. The luciferase activities for COS-7 cells were measured using the Dual-luciferase reporter assay system (Promega). Mouse mammary tumour virus promoter in pGL3-Basic (a gift from Professor J. Palvimo, University of Kuopio, Kuopio, Finland) was used as a control for androgen response.

Quantitative reverse transcription-polymerase chain reaction. Total RNA from LNCaP cells was isolated with TRIzol reagent (Invitrogen-Gibco) for quantitative reverse transcriptionpolymerase chain reaction (RT-PCR) measurements, according to the manufacturer's protocol. RNA isolated from prostate samples was also used. The first-strand cDNA was synthesized with the First-Strand cDNA synthesis kit (Amersham Biosciences, Piscataway, NJ, USA) using $1 \mu \mathrm{g}$ of RNA and $\operatorname{pd}(\mathrm{N}) 6$ random hex deoxynucleotides according to the manufacturer's instructions. mRNA levels for LNCaP cells and human prostate samples were measured by quantitative RT-PCR analysis (ABI 7700, Applied Biosystems, Foster City, CA, USA) as described previously (10). Forward and reverse primers for SORD mRNA detection were 5'-TGAGA TGACCACCGTACCCC-3' and 5'-GAAACACGCCCTTG ATATCCA-3', respectively. The amplicon was detected using fluorogenic probe 5'-FAM-TGCATGCAGCCATCCGG GAG-TAMRA-3'. The primers and the probe for the $18 \mathrm{~S}$ amplicon were 5'-TGGTTGCAAAGCTGAAACTTAAAG-3', 5'-AGTCAAATTAAGCCGCAGGC-3' and 5'-VIC-CCTGG TGGTGCCCTTCCGTCA-TAMRA-3', respectively. 
Table I. GeneChip array results of some known androgen-regulated genes, prostate cancer associated genes and SORD in three castrated samples and in three benign prostate samples.

\begin{tabular}{|c|c|c|c|c|c|c|c|c|}
\hline \multirow{2}{*}{$\begin{array}{l}\text { Gene } \\
\text { symbol } \\
D P P 4\end{array}$} & \multirow{2}{*}{$\frac{\text { Gene name }}{\text { Dipeptidyl-peptidase } 4 \text { (CD26) }}$} & \multicolumn{3}{|c|}{$\begin{array}{c}\text { Expression in } \\
\text { castrated samples }\end{array}$} & \multicolumn{3}{|c|}{$\begin{array}{l}\text { Expression in benign } \\
\text { prostate samples }\end{array}$} & \multirow{2}{*}{$\begin{array}{c}\begin{array}{c}\text { Fold change } \\
\text { in expression }\end{array} \\
2.81\end{array}$} \\
\hline & & 6.53 & 6.07 & 6.53 & 9.9 & 7.67 & 10 & \\
\hline$M S M B$ & Microseminoprotein, $ß$ & 11.79 & 10.85 & 10.43 & 13.58 & 14.01 & 14.2 & 2.91 \\
\hline$S O R D$ & Sorbitol dehydrogenase & 8.12 & 8.02 & 8.1 & 10.53 & 9.81 & 10.84 & 2.31 \\
\hline$A Z G P 1$ & $\alpha$-2-glycoprotein 1 , zinc-binding & 9.6 & 9.16 & 8.36 & 12.12 & 9.57 & 12 & 2.19 \\
\hline$K L K 2$ & Kallikrein-related peptidase 2 & 10.82 & 9.5 & 11.29 & 12.7 & 12.31 & 13.06 & 2.15 \\
\hline TMPRSS2 & Transmembrane protease, serine 2 & 8.25 & 7.94 & 9.81 & 11.46 & 10.13 & 11.22 & 2.27 \\
\hline CYP3A5 & Cytochrome P450, subtype 3 A5 & 7.58 & 6.69 & 5.21 & 9.03 & 8.34 & 9.28 & 2.39 \\
\hline$K L K 4$ & Kallikrein-related peptidase 4 & 6.81 & 6.33 & 7.56 & 9.81 & 7.77 & 8.56 & 1.81 \\
\hline$K L K 3$ & Prostate-specific antigen & 12.06 & 11.08 & 12.87 & 13.81 & 13.57 & 13.96 & 1.78 \\
\hline
\end{tabular}

Normalized, $\log 2$-transformed expression values are presented. Fold expression change was calculated from means of log2-transformed expression values between castrated samples and benign prostate samples.

Immunochemistry. Proteins from snap-frozen prostate tissue were isolated using RIPA buffer $(25 \mathrm{mM}$ Tris- $\mathrm{HCl} \mathrm{pH} \mathrm{7.6,}$ $150 \mathrm{mM} \mathrm{NaCl}, 1 \% \mathrm{NP}-40,1 \%$ sodium deoxycholate, $0.1 \%$ SDS) as described (11). After boiling the samples in reducing SDS sample buffer for $5 \mathrm{~min}, 10 \mu \mathrm{g}$ of protein was loaded per lane and the samples were electrophoresed on $10 \%$ polyacrylamide SDS gel and transferred to a PVDF membrane. SORD was detected with SORD monoclonal antibody (M01, clone 4D3, catalogue no. H00006652-M01, Abnova, Taipei City, Taiwan, dilution 1:10000). For negative control, a 10-fold excess of SORD recombinant protein (H00006652-Q01, Abnova) was incubated with antibody overnight at $4^{\circ} \mathrm{C}$ before detection. Binding of target proteins on the membranes was revealed with goat anti-mouse HRP-conjugated secondary antibodies (Santa Cruz Biotechnology, Santa Cruz, CA, USA). The protein bands were visualised by chemiluminescence.

Multitissue block slides containing $4 \mu \mathrm{m}$ sections of formalin-fixed, paraffin-embedded prostate samples were used for immunohistochemistry. The sections were then routinely deparaffinised in xylene and rehydrated in descending ethanol series. To enhance immunoreactivity, the sections were incubated in a citrate buffer ( $\mathrm{pH}$ 6.0) and boiled. Endogenous peroxidase activity was eliminated by incubation in hydrogen peroxide and absolute methanol. SORD monoclonal antibody (M01, clone 4D3, Abnova, dilution 1:7000) was used. The Envision-kit (Dako, Glostrup, Denmark) was used for visualisation. The immunostainings were classified as negative, weakly positive or strongly positive. Using these criteria, the immunostaining results were evaluated by two observers (P.H. and M.H.V.) and a consensus was reached.

Chromatin immunoprecipitation (ChIP). ChIP was carried out in the conditions described previously (12) using a commercially available SimpleChIP kit (Cell Signaling Technology, Danvers, MA, USA). Androgen receptor antibody PG-21 (catalogue no. 06-680, Millipore, Temecula, CA, USA) was used in the immunoprecipitation reaction. The input genomic DNA samples were treated in the same way except that no immunoprecipitation was performed. Primers 5'-CCTATTTGGCCATCTTGGAA-3' and 5'-CTTGCCCGC TTGATTACCTA-3' were used in PCR reactions to amplify the region of the predicted androgen receptor binding site at the SORD promoter. To achieve linear amplification ranges, immunoprecipitated and input products were amplified with 40 and 36 cycles, respectively.

Statistical analyses. Student's t-test was used for comparison between two groups and the difference was considered to be statistically significant when the P-value was $<0.05$. The immunostaining quantification results were analyzed using SPSS for Windows 16.0 (SPSS Inc., Chicago, IL, USA).

\section{Results}

Androgen regulation of SORD $m R N A$ in human prostate tissue and the LNCaP prostate cancer cell line. In an attempt to find novel androgen-regulated genes in the human prostate, we analysed relative gene expression levels in benign prostate tissue samples and in prostate samples taken after castration using a GeneChip array. Normalised $\log 2$-transformed expression values of SORD mRNA (Affymetrix cell ID: 201563_at) in three benign prostate tissue samples and three prostate samples taken after castration were 10.53, 9.81, $10.84,8.12,8.02$ and 8.10 , respectively. This provided a 2.31-fold change in expression when comparing the three benign prostate tissue samples to the three castrated prostate tissue samples. The normalised $\log 2$-transformed expression values of SORD and some known androgen-regulated genes and prostate cancer associated genes identified here are presented in Table I.

SORD expression and androgen regulation were then analysed in the LNCaP cell line using quantitative RT-PCR. A 7.5-fold increase in SORD mRNA expression was detected after $48 \mathrm{~h}$ of treatment with $10 \mathrm{nM} \mathrm{R} 1881$ (Fig. 1). 


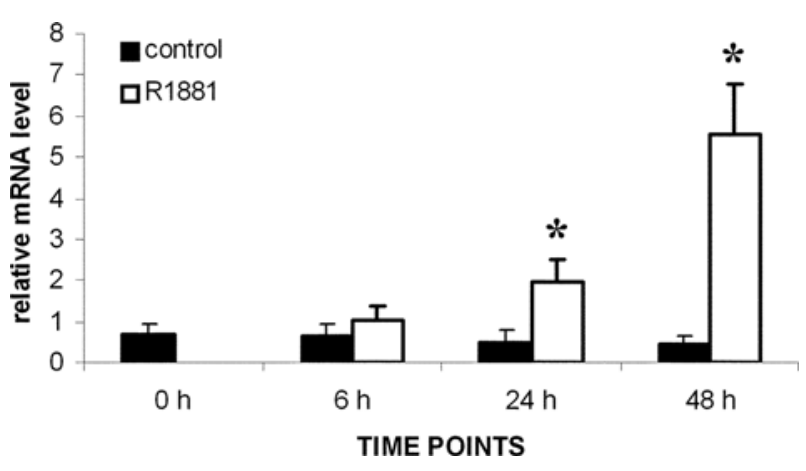

Figure 1. Response of SORD mRNA in LNCaP cells after treatment with $10 \mathrm{nM}$ synthetic androgen R1881. A clear statistically significant induction of SORD mRNA was seen after 24 and 48 h. ${ }^{*} \mathrm{P}<0.001$ Student's paired samples t-test, compared with $48 \mathrm{~h}$ control. The values represent means \pm standard deviations of 5 individual samples.

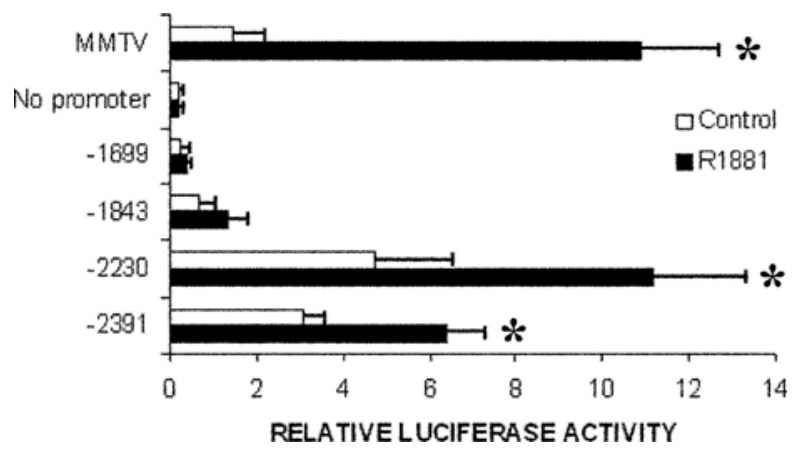

Figure 2. Results from transient transfections of different SORD promoter constructs into COS-7 cells together with an AR expression vector. Statistically significant induction of luciferase activity by $100 \mathrm{nM}$ R1881 was detected with constructs -2391 to +101 and -2230 to +101 . With shorter constructs no induction was seen. Mouse mammary tumour virus promoter (MMTV) in pGL3-Basic was used as the positive control. *Statistically significant induction of luciferase activity by R1881 compared with the respective control, $\mathrm{P}<0.05$ Student's paired samples t-test. The values represent means \pm standard errors of means of 4 samples in at least three independent experiments.

Identification of region for putative androgen receptor binding in SORD promoter. To study the involvement of transcriptional regulation in the regulation of SORD by androgens and to locate the putative androgen-responsive regulatory region, a series of constructs containing the SORD promoter region starting from positions $-2391,-2230,-1843$ or -1699 to +101 (positions numbered from the transcription initiation start site) were transfected into COS-7 cells together with an AR expression vector and the cells were treated with androgen or vehicle only. The results for transient transfections are shown in Fig. 2. Androgen induction was detected for the constructs -2391 to +101 and -2230 to +101 , but not for the constructs with shorter promoter regions, indicating that the promoter region -2230 to -1843 contains sequence elements critical for SORD androgen induction. This region was screened for putative transcription binding DNA sequences using the TESS programme. Transcription factor search did not reveal any potential AR binding sites in the SORD 5'

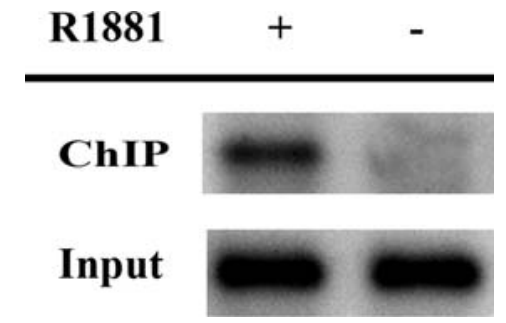

Figure 3. Experimental verification of putative androgen receptor binding site. LNCaP cells were treated in the presence or absence of $3.3 \mathrm{nM}$ of synthetic androgen R1881. Primers 5'-CCTATTTGGCCATCTTGGAA-3' and 5'-CTTGCCCGCTTGATTACCTA-3' were used in PCR reactions to amplify the region containing the predicted androgen receptor binding site at the SORD promoter. To achieve linear amplification ranges, immunoprecipitated and input products were amplified with 40 and 36 cycles, respectively. The PCR products of sample DNA after AR immunoprecipitation (ChIP) or after similar treatment with no immunoprecipitation (Input) are shown.

promoter between positions -2230 and -1843 . However, several potential glucocorticoid receptor binding sequences were identified at positions -2179 to $-2173,-1986$ to -1980 , -1974 to -1968 and -1853 to -1847 at the SORD 5' promoter. As the AR and glucocorticoid receptor can activate the same hormone response element (13), these regions were regarded as potential androgen receptor binding sites. These potential binding sites were screened using ChIP (data not shown), and immunoprecipitation with the AR antibody was demonstrated between positions -1943 and -1824 (Fig. 3). Thus, the glucocorticoid receptor binding sequence at position -1853 to -1847 may serve as an androgen receptor binding site as well.

SORD protein expression in the human prostate. SORD protein was detected in a prostate sample using Western blotting. Incubation with blocking SORD protein led to the loss of specific binding in the Western blotting as expected (Fig. 4A). The expression and localisation of SORD in the prostate was further studied immunohistochemically (Fig. 4B-C). The SORD antibody strongly stained the luminal epithelial cells in benign glands, but there was no staining in the prostatic stroma. In prostate cancer samples, the expression was also localised in epithelial cells. In some cases, nuclear staining was present (Fig. 4B). The significance of this nuclear staining remains unclear, but it is likely to be unspecific staining. The immunostaining was evaluated using the criteria specified in Materials and methods to quantify the expression of SORD. In prostate cancer, there were $1(4 \%), 10(40 \%)$ and 14 $(56 \%)$ samples with negative, weakly positive and strongly positive expressions, respectively. In benign prostatic glands (BPH samples and benign structures in prostate cancer samples), there were $0(0 \%), 5(33 \%)$ and $10(67 \%)$ samples with negative, weakly positive and strongly positive expressions, respectively. In hormone naive patients (BPH patients and prostate cancer patients), there were strong expressions of SORD in benign glands in 9/13 (69.2\%) cases, whereas in prostate cancer the expression was strong in 5/16 (31.2\%) cases. Interestingly, in castrated patients there was strong SORD expression in all prostate cancer cases (Table II). In prostate cancer, strong SORD expression was further detected 

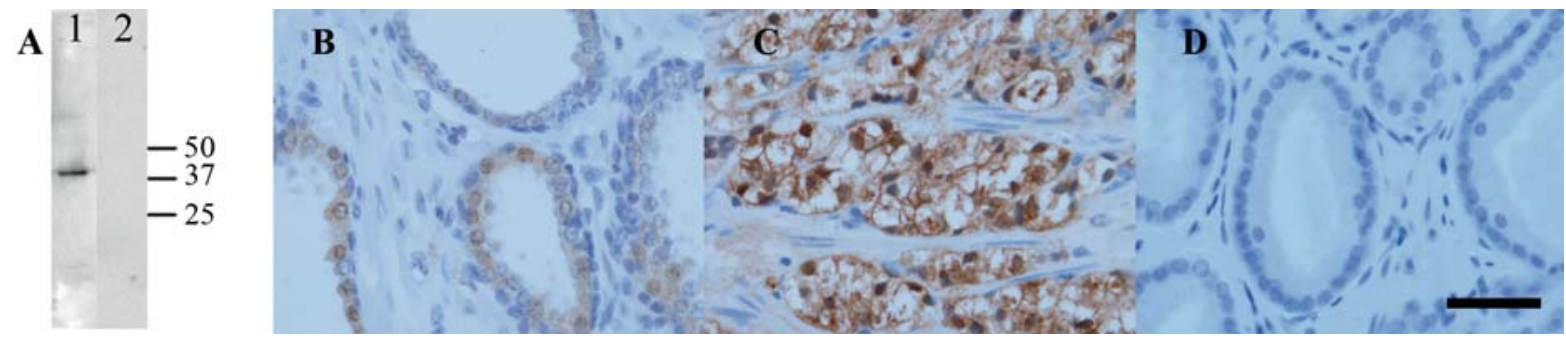

Figure 4. SORD protein expression in the human prostate. Western blotting of prostate protein sample with SORD antibody detected $\sim 37$ kDa protein, as expected (A, lane 1). Antibody blocking with SORD recombinant protein abolishes the specific binding of the SORD antibody (A, lane 2). The reaction conditions were identical in experiments presented in (A), lanes 1 and 2, except added SORD protein for lane 2. Protein ladder with respective molecular weights is marked on the right side of (A). Immunohistochemistry of prostate tissue slides detected SORD protein in the luminal epithelial cells of normal prostate (B) and in Gleason pattern 5 adenocarcinoma (C). No staining was seen in the stroma (B). Negative control, normal prostate immunohistochemistry with PBS instead of SORD primary antibody (D). Primary antibody dilution 1:7000 (B and C). The black bar in right lower corner of (D) corresponds to 50 $\mu \mathrm{m}$, same magnification in (B-D).

Table II. Immunostaining of SORD in benign prostate and prostate cancer.

\begin{tabular}{|c|c|c|c|}
\hline & Hormone naive patients, $\mathrm{n}(\%)$ & Castrated patients, n (\%) & P-value \\
\hline Prostate cancer & & & 0.002 \\
\hline \multicolumn{4}{|c|}{ SORD expression level } \\
\hline Negative & $1 \quad(6.2)$ & $0 \quad(0)$ & \\
\hline Weakly positive & $10(62.5)$ & $0 \quad(0)$ & \\
\hline Strongly positive & $5(31.2)$ & $9(100)$ & \\
\hline Benign glands & & & 1.00 \\
\hline \multicolumn{4}{|c|}{ SORD expression level } \\
\hline Negative & $0 \quad(0)$ & $0 \quad(0)$ & \\
\hline Weakly positive & $4(30.8)$ & $1 \quad(50)$ & \\
\hline Strongly positive & $9(69.2)$ & $1 \quad(50)$ & \\
\hline
\end{tabular}

The immunostaining of SORD was increased in prostate cancer patients with chemical or surgical castration as a treatment compared with hormone naive patients. Significant differences were not detected in the immunostaining at benign glands (Fisher's exact test).

Table III. The association of SORD immunostaining with Gleason patterns and PSA concentrations in prostate cancer and in benign glands of prostate cancer samples.

\begin{tabular}{|c|c|c|c|c|c|c|}
\hline & \multicolumn{5}{|c|}{ SORD immunostaining } & \multirow[b]{3}{*}{ P-value } \\
\hline & \multicolumn{2}{|c|}{ Prostate cancer } & \multirow[b]{2}{*}{ P-value } & \multicolumn{2}{|c|}{ Benign glands } & \\
\hline & Weakly positive & Strongly positive & & Weakly positive & Strongly positive & \\
\hline Gleason pattern & $3.0 \quad(2.0-3.0)$ & $4.0 \quad(3.0-5.0)$ & 0.049 & $2.5 \quad(2.0-4.0)$ & $3.0 \quad(2.0-3.5)$ & 0.826 \\
\hline PSA (ng/ml) & $6.4(3.4-10.0)$ & $19.0(7.3-142.5)$ & 0.029 & $5.9(3.2-11.0)$ & $8.2(3.6-19.5)$ & 0.461 \\
\hline
\end{tabular}

in patients with higher Gleason patterns and higher PSA concentrations compared with patients with weak SORD expressions (Table III). No such similarity was detected in the expression of SORD in benign glands of the same prostates (Table III), meaning the elevated expression of SORD was connected to prostate cancer. 


\section{Discussion}

During the search for novel androgen-regulated genes in prostate, we identified SORD. SORD is an enzyme that is involved in glucose metabolism, converting sorbitol to fructose using $\mathrm{NAD}(+)$ as a coenzyme. In the reproductive tract, SORD is believed to be involved in energy production for spermatozoa, due to the high glucose and fructose content in semen (14). Seminal vesicles and ampuls of vas deferences are the main sources of fructose in semen (15). SORD is widely expressed in human tissues (3) and therefore it may have other tissue-specific functions besides converting sorbitol to fructose, as fructose production may not be required in every tissue in which SORD is expressed.

To our knowledge, the hormonal regulation of SORD expression has not been described in humans. However, dexamethasone administration increases serum SORD levels in rats (16). It is unclear whether glucocorticoid-induced elevated SORD levels in rats are a consequence of the steroid hormone up-regulated expression of SORD or liver tissue damage (16). Here, we detected decreased expressions of SORD mRNA in the prostate after castration compared with benign prostate tissue without castration. Furthermore, SORD expression was high in prostate cancer with high Gleason patterns and high PSAs. This association was absent in benign glands of cancer samples, suggesting a cancer-specific elevated expression of SORD. The expression of SORD was also high in patients with chemical or surgical castration. The castrated prostate cancer patients had hormone refractory prostate cancer with mainly high PSAs and high Gleason scores. This supports the similar regulation of SORD and PSA in prostate cancer because the serum concentrations of androgen-regulated PSA were high and the tissue expression of SORD was high.

Janus kinase (JAK) and signal transducer and activator of transcription (STAT) pathway is linked to prostate cancer. STAT5A/B regulates the viability of prostate cancer cells and is activated in human prostate cancer cells (17). Patients with specific STAT3 phosphorylation had a different prognosis compared to patients without this STAT3 phosphorylation (18). Furthermore, STAT3 is involved in the metastatic behaviour of human prostate cancer cells (19). In the heart, the aldose reductase pathway has been demonstrated to be a key component of myocardial reperfusion injury (20). JAK2 and STAT5 signalling induction were detected after global ischaemia. Pharmacological inhibition of sorbitol dehydrogenase blocks JAK2 and STAT5 activation in rat heart (21). The role of SORD in regulation of the JAK-STAT pathway in the human prostate remains to be clarified, but it might be a potential physiological role for SORD in the prostate.

We have demonstrated the androgen-regulated expression of SORD in the human prostate and identified a putative androgen responsive regulatory region at the $5^{1}$ promoter of $S O R D$. The function of SORD in the prostate is unknown because the majority of fructose production seems to occur in the reproductive tract upstream of the prostate (14). However, as the expression of SORD is regulated by androgens, it is logical to hypothesise that SORD also has a relevant function in the prostate. Furthermore, the expression of SORD was localised in the epithelial cells of both benign and malignant prostate tissue, which means that SORD is expressed in the most physiologically active cells of the prostate.

The expression of SORD is regulated by androgens in human prostate. In prostate cancer, increased immunostaining was associated with high Gleason patterns and high serum PSA concentrations. Our results suggest the need for more detailed functional characterisation of SORD in the human prostate as well as evaluation of the prognostic significance of SORD in larger clinical materials.

\section{Acknowledgements}

We are grateful to Ms. Mirja Mäkeläinen for technical assistance, Mrs. Erja Tomperi and Mrs. Mirja Vahera for carrying out the immunohistochemistry, and Mrs. Marja Tolppanen for help in cell culturing. Dr Mika Ilves performed the quantitative RT-PCR experiments. Dr Jussi Vuoristo performed the GeneChip arrays. Pasi Ohtonen, M.Sc., assisted with statistical analyses. This study was supported by grants from the Päivikki and Sakari Sohlberg Foundation, the Finnish Medical Fund and the Cancer Society of Northern Finland.

\section{References}

1. Shin YS, Rieth M, Endres W and Haas P: Sorbitol dehydrogenase deficiency in a family with congenital cataracts. J Inherit Metab Dis 7 (Suppl. 2): S151-S152, 1984.

2. Iwata T, Popescu NC, Zimonjic DB, Karlsson C, Hoog JO, Vaca G, Rodriguez IR and Carper D: Structural organization of the human sorbitol dehydrogenase gene (SORD). Genomics 26: 55-62, 1995.

3. Carr IM and Markham AF: Molecular genetic analysis of the human sorbitol dehydrogenase gene. Mamm Genome 6: 645-652, 1995.

4. El-Kabbani O, Darmanin C and Chung RP: Sorbitol dehydrogenase: structure, function and ligand design. Curr Med Chem 11: 465-476, 2004.

5. Frenette G, Thabet M and Sullivan R: Polyol pathway in human epididymis and semen. J Androl 27: 233-239, 2006.

6. Knudsen KE, Arden KC and Cavenee WK: Multiple G1 regulatory elements control the androgen-dependent proliferation of prostatic carcinoma cells. J Biol Chem 273: 20213-20222, 1998.

7. Gelmann EP: Molecular biology of the androgen receptor. J Clin Oncol 20: 3001-3015, 2002.

8. Heinlein CA and Chang C: Androgen receptor (AR) coregulators: an overview. Endocr Rev 23: 175-200, 2002.

9. Moilanen AM, Hakkola J, Vaarala MH, Kauppila S, Hirvikoski P, Vuoristo JT, Edwards RJ and Paavonen TK: Characterization of androgen-regulated expression of CYP3A5 in human prostate. Carcinogenesis 28: 916-921, 2007.

10. Majalahti-Palviainen T, Hirvinen M, Tervonen V, Ilves M, Ruskoaho $\mathrm{H}$ and Vuolteenaho O: Gene structure of a new cardiac peptide hormone: a model for heart-specific gene expression. Endocrinology 141: 731-740, 2000.

11. Sefton BM: Labeling cultured cells with $32 \mathrm{Pi}$ and preparing cell lysates for immunoprecipitation. In: Current Protocols in Molecular Biology. Ausubel FM, Brent R, Kingston RE, Moore DD, Seidman JG, Smith JA and Struhl K (eds). John Wiley \& Sons Inc., Unit 18.2, 2005.

12. Masuda K, Werner T, Maheshwari S, Frisch M, Oh S, Petrovics G, May K, Srikantan V, Srivastava S and Dobi A: Androgen receptor binding sites identified by a GREF_GATA model. J Mol Biol 353: 763-771, 2005.

13. Beato M: Gene regulation by steroid hormones. Cell 56: 335-344, 1989.

14. Mann T: Studies on the metabolism of semen: 3. Fructose as a normal constituent of seminal plasma. Site of formation and function of fructose in semen. Biochem J 40: 481-491, 1946.

15. Mann T and Lutwak-Mann C: Evaluation of the functional state of male accessory glands by the analysis of seminal plasma. Andrologia 8: 237-242, 1976. 
16. Jackson ER, Kilroy C, Joslin DL, Schomaker SJ, PruimboomBrees I and Amacher DE: The early effects of short-term dexamethasone administration on hepatic and serum alanine aminotransferase in the rat. Drug Chem Toxicol 31: 427-445, 2008.

17. Tan SH and Nevalainen MT: Signal transducer and activator of transcription 5A/B in prostate and breast cancers. Endocr Relat Cancer 15: 367-390, 2008.

18. Tam L, McGlynn LM, Traynor P, Mukherjee R, Bartlett JM and Edwards J: Expression levels of the JAK/STAT pathway in the transition from hormone-sensitive to hormone-refractory prostate cancer. Br J Cancer 97: 378-383, 2007.

19. Abdulghani J, Gu L, Dagvadorj A, Lutz J, Leiby B, Bonuccelli G, Lisanti MP, Zellweger T, Alanen K, Mirtti T, Visakorpi T, Bubendorf L and Nevalainen MT: Stat3 promotes metastatic progression of prostate cancer. Am J Pathol 172: 1717-1728, 2008 .
20. Hwang YC, Kaneko M, Bakr S, Liao H, Lu Y, Lewis ER, Yan S, Ii S, Itakura M, Rui L, Skopicki H, Homma S, Schmidt AM, Oates PJ, Szabolcs M and Ramasamy R: Central role for aldose reductase pathway in myocardial ischemic injury. FASEB J 18: 1192-1199, 2004

21. Hwang YC, Shaw S, Kaneko M, Redd H, Marrero MB and Ramasamy R: Aldose reductase pathway mediates JAK-STAT signaling: a novel axis in myocardial ischemic injury. FASEB J 19: 795-797, 2005. 